Proyecciones Journal of Mathematics

Vol. 40, No 6, pp. 1683-1691, December 2021.

Universidad Católica del Norte

Antofagasta - Chile

\title{
The structure of Cayley graphs of dihedral groups of Valencies 1, 2 and 3.
}

\author{
Saba AL-Kaseasbeh \\ Tafila Technical University, Jordan \\ Ahmad Erfanian \\ Ferdowsi University of Mashhad, Iran \\ Received: August 2020. Accepted : November 2021
}

\begin{abstract}
Let $G$ be a group and $S$ be a subset of $G$ such that $e \notin S$ and $S^{-1} \subseteq$ $S$. Then $\operatorname{Cay}(G, S)$ is a simple undirected Cayley graph whose vertices are all elements of $G$ and two vertices $x$ and $y$ are adjacent if and only if $x y^{-1} \in S$. The size of subset $S$ is called the valency of $\operatorname{Cay}(G, S)$. In this paper, we determined the structure of all Cay $\left(D_{2 n}, S\right)$, where $D_{2 n}$ is a dihedral group of order $2 n, n \geq 3$ and $|S|=1,2$ or 3 .
\end{abstract}

Keywords: Valency, Cayley graph, Dihedral group.

2010 Mathematics Subject Classification: 05C25. 


\section{Introduction}

Algebraic graph theory is one of the most important branches of mathematics, playing an essential role in other mathematical fields in which algebraic methods are applied to problems about graphs.

In recent years much attention has been paid to associate a graph with an algebraic object. The properties of the group are employed to investigate a graph invariant and vice versa. One very old and important such example that highlights the interplay between finite groups and graph theory is the notation of a Cayley graph. The definition of the Cayley graph was first introduced by Arthur Cayley in 1878 [1]. In the last 50 years, there has been a great deal of interest in the theory of Cayley graphs which has played an essential role in algebraic graph theory. It is related to problems in group theory and graph theory such as classification, group and graph isomorphisms, varieties of graph coloring, diameter problems and enumeration problems, (see [7] and [2]). Also, many interesting application of Cayley graph in computer science and biological science, for instance (see [3] and [6]). There are also some important topics of graph theory and group theory in the Cayley graphs of dihedral groups. For instance integrality [8], distance-reqular [9], locally primitive [10], degree diameter problem [4] and edge transitivity [5] of $\operatorname{Cay}\left(D_{2 n}, S\right)$. In this paper, we aim to give the graph structure of $\operatorname{Cay}\left(D_{2 n}, S\right)$ for $n \geq 3$ and $|S|=1,2$ or 3 .

The terminology and notations used in this paper are standered. For example for a positive integer $n$, we use $Z_{n}$ and $D_{2 n}$ to denote the cyclic group of order $n$ and dihedral group of order $2 n$, respectively. In fact they have the presentation $Z_{n}=<x \mid x^{n}=e>=\left\{e, x, x^{2}, \cdots, x^{n-1}\right\}$ and $D_{2 n}=<$ $a, b \mid a^{n}=b^{2}=e, b a b=a^{-1}>=\left\{e, a, a^{2}, \cdots, a^{n-1}, b, a b, \cdots, a^{n-1} b\right\}$. For a graph $X$ the set of vertices and edges are denoted by $V(G)$ and $E(G)$, respectively. For two vertices $x, y \in V(G)$, we denote $x \sim y$ or $x-y$ if $x$ and $y$ are adjacent. A graph with no edge is called an empty graph. The complement of $X$, denoted by $\bar{X}$, is a graph such that $V(X)=V(\bar{X})$ and two vertices are adjacent in $\bar{X}$ if and only if there are not adjacent in $X$. The degree of vertex $x \in V(G)$ denoted by $\operatorname{deg}(x)$, is the number of adjacent vertices of $x$. We denote by $K_{n}, P_{n}$ and $C_{n}$ the complete graph, the path graph and the cycle graph with $\mathrm{n}$ vertices, respectively. The union of two graphs $X_{1}$ and $X_{2}$ denoted by $X_{1} \cup X_{2}$ is a graph with $V\left(X_{1} \cup X_{2}\right)=V\left(X_{1}\right) \cup V\left(X_{2}\right)$ and $E\left(X_{1} \cup X_{2}\right)=E\left(X_{1}\right) \cup E\left(X_{2}\right)$. If $X_{1}=X_{2}$, then $X_{1} \cup X_{1}$ will denote by $2 X_{1}$ and similarly $n X_{1}$ stands for the union of $n$ copies of $X_{1}$. Moreover, 
the Cartesian product of two graphs $X_{1}$ and $X_{2}$ denoted by $X_{1} \square X_{2}$ is a graph with vertex set $V\left(X_{1} \square X_{2}\right)=\left\{(x, y) \mid x \in V\left(X_{1}\right), y \in V\left(X_{2}\right)\right\}$. Two vertices $\left(x_{1}, y_{1}\right)$ and $\left(x_{2}, y_{2}\right)$ are adjacent if and only if $x_{1}=x_{2}$ and $y_{1}$ is adjacent to $y_{2}$ in $X_{2}$ or $y_{1}=y_{2}$ and $x_{1}$ adjacent to $x_{2}$ in $X_{1}$. For a given group $G$, the Cayley graph of $G$ with respect to a subset $S$ of $G$, denoted by $\operatorname{Cay}(G, S)$ is an undirect simple graph whose vertices are all elements of $G$ where $e \notin S$ and $S^{-1} \subseteq S$. Two vertices $x$ and $y$ are adjacent if and only if $x y^{-1} \in S$. The size of subset $S$ is called the valency of the Cayley graph. Some of known properties of $\operatorname{Cay}(G, S)$ are $|S|$-regular, vertex transitive (automorphism group of the $\operatorname{Cay}(G, S)$ acts transitively on $V(G)$ and connected whenever $S$ is a generating set. More background information on graph theory can be found in [12]. For the group theoretical concepts not defined here, we refer the reader to [11].

\section{Cayley Graphs of Dihedral Groups of Valencies 1 and 2.}

In this section, we investigate and determine the Cayley graphs $\Gamma=\operatorname{Cay}(G, S)$ on a dihedral group $D_{2 n}$ of valency 1 and 2 . Let us start with the case that $|S|=1$.

Theorem 2.1. Let $D_{2 n}$ be a dihedral group of order $2 n$. If $S \subseteq D_{2 n}$ such that $e \notin S, S^{-1}=S$ and $|S|=1$. Then $\operatorname{Cay}\left(D_{2 n}, S\right)=n K_{2}$.

Proof. Assume that $S=\{x\}$, then by given definition of $S$, we should have $x \neq e$ and $x^{-1}=x$. Thus, $x^{2}=e$. Now, assume that $g \in D_{2 n}$ is an arbitrary element such that $g \neq x$. Then we can see that $x g$ is adjacent to $g$, since, $(x g) g^{-1}=x g g^{-1}=x \in S$. Now, consider the cyclic subgroup $H=\langle x\rangle=\{e, x\}$ of $D_{2 n}$, where $|H|=2$. It tends out that $\left[D_{2 n}: H\right]=n$. Therefore there are $n$ distinct right cosets $H g_{1}=H, H g_{2}, \cdots, H g_{n}$, where $g_{1}=e \in H$ and $g_{2}, g_{3}, \cdots, g_{n} \notin H$. Moreover, we have $D_{2 n}=H g_{1} \cup$ $H g_{2} \cdots \cup H g_{n}=\{e, x\} \cup\left\{g_{2}, x g_{2}\right\} \cup\left\{g_{3}, x g_{3}\right\} \cup \cdots \cup\left\{g_{n}, x g_{n}\right\}$. Hence, there are $n$ edges $e-x, g_{2}-x g_{2}, \cdots g_{n}-x g_{n}$ and this concludes the proof.

As an easy example, we can see that $\operatorname{Cay}\left(D_{8}, S\right)$, where $S=\{a\}$ is a graph consisting of 4 edges $a-e, a^{2}-a^{3}, b-a b, a^{2} b-a^{3} b$. In other words, $\operatorname{Cay}\left(D_{8}, S\right)=4 K_{2}$. Now, we consider $\operatorname{Cay}\left(D_{2 n}, S\right)$, such that $|S|=2$. According to conditions $e \notin S, S^{-1}=S$, we can see that $S=\left\{x, x^{-1}\right\}$ whenever $x \neq e, x^{2} \neq e$ or $S=\{x, y\}$, where $x \neq e, y \neq e$ and $x^{2}=y^{2}=e$. In the following theorem, we give the graph structure of $\operatorname{Cay}\left(D_{2 n}, S\right)$, where $S=\left\{x, x^{-1}\right\}$ and $x \neq x^{-1}$. 
Theorem 2.2. Let $D_{2 n}$ be a dihedral group of order $2 n$. If $S \subseteq D_{2 n}$ such that $S=\left\{x, x^{-1}\right\}$, where $x \neq x^{-1}$ and $o(x)=m$, then $\operatorname{Cay}\left(D_{2 n}, S\right)=$ $\frac{2 n}{m} C_{m}$.

Proof. Suppose that $D_{2 n}=\left\{e, a, a^{2}, a^{3}, \cdots, a^{n-1}, b, a b, a^{2} b, \cdots, a^{n-1} b\right\}$. Since $x \neq x^{-1}$, we have $o(x) \neq 2$. Also, we know that $o\left(a^{i} b\right)=2$, for $i=0,1,2, \cdots, n-1$. Therefore, $x=a^{i}$, for some $i$, where $i=$ $1,2, \cdots, n-1$ and when $n$ is even $i \neq \frac{n}{2}$. Now, assume that $n$ is odd, then $x=a^{i}$, where $i=1,2, \cdots, n-1$. We claim that the following is a cycle of length $\mathrm{m} e-x-x^{2}-x^{3} \cdots-x^{m-1}-x^{m}=e$. Notice that $\left(x^{k}\right)\left(x^{k+1}\right)^{-1}=a^{i k} a^{-i k-i}=a^{-i}=x^{-1} \in S$, for $k=0,1,2, \cdots m$. Moreover, let $H=\langle x\rangle=\left\{e, x, \cdots, x^{m-1}\right\}$ be a cyclic subgroup of $D_{2 n}$ of order $\mathrm{m}$, then we have $\left[D_{2 n}: H\right]=\frac{2 n}{m}=t$. Thus there are $t$ distinct right cosets $H g_{1}=H, H g_{2}, \cdots, H g_{t}$, such that $g_{1}=e \in H$ and $g_{2}, g_{3}, \cdots, g_{t} \notin H$. It tends out for each right coset $H g_{j}=\left\{g_{j}, x g_{j}, x^{2} g_{j}, \cdots, x^{m-1} g_{j}\right\}$, we have a cycle $g_{j}-x g_{j}-x^{2} g_{j}-\cdots-x^{m-1} g_{j}-x^{m} g_{j}=g_{j}$, for $j=1,2, \cdots, t$. Therefore, we have $t$ cycles of length $m$. Hence $\operatorname{Cay}\left(D_{2 n}, S\right)=t C_{m}=$ $\frac{2 n}{m} C_{m}$. When $n$ is even, then $x=a^{\frac{n}{2}}$ has order 2 and does not satisfy in our assumption.

In the next theorem, we deal with the second case for $S$. Take $S=\{x, y\}$ such that $x^{2}=y^{2}=e$.

Theorem 2.3. Let $D_{2 n}$ be a dihedral group of order $2 n$ and $S \subseteq D_{2 n}$ such that $S=\{x, y\}$, where $x^{2}=y^{2}=e$. Then $\operatorname{Cay}\left(D_{2 n}, S\right)=\frac{n}{m} C_{2 m}$ where $m=o(x y)$.

Proof. Consider the case where $\mathrm{n}$ is odd. Since $o(x)=o(y)=2$, then $x$ and $y$ cannot be of the form $a^{i}$, where $i=1,2, \cdots, n-1$. Thus $x=a^{i} b$ and $y=a^{j} b$, where $1 \leq i \neq j \leq n-1$. Now, since $\left[y(x y)^{k}\right]\left[(x y)^{k+1}\right]^{-1}=$ $y(x y)^{k}(x y)^{-k-1}=y(x y)^{-1}=y y^{-1} x=x \in S$ and

$\left[(x y)^{k}\right]\left[y(x y)^{k}\right]^{-1}=(x y)^{k}(x y)^{-k} y^{-1}=y^{-1}=y \in S$, we have the following cycle of length $2 m, e-y-x y-y(x y)-(x y)^{2}-y(x y)^{2}-(x y)^{3}-$ $\cdots-y(x y)^{m-2}-(x y)^{m-1}-y(x y)^{m-1}-(x y)^{m}=e$. We define the following subgroup $D_{2 m}=<(x y), y \mid(x y)^{m}=y^{2}=e, y(x y) y=(x y)^{-1}>=$ $\left\{e,(x y),(x y)^{2}, \cdots\right.$, $\left.(x y)^{m-1}, y, y(x y), \cdots, y(x y)^{m-1}\right\}$. As above, we have a cycle of length $2 m$ between all elements of $D_{2 m}$. On the other hand,

$\left[D_{2 n}: D_{2 m}\right]=\frac{2 n}{2 m}=\frac{n}{m}=t$. Therefore, we have $D_{2 n}=D_{2 m} \cup D_{2 m} g_{2} \cup$ $D_{2 m} g_{3} \cup \cdots \cup D_{2 m} g_{t}$, where each $D_{2 m} g_{k}$ consists of a cycle of length $2 m$ as 
the following

$g_{k}-y g_{k}-(x y) g_{k}-y(x y) g_{k}-(x y)^{2} g_{k}-\cdots-y(x y)^{m-1} g_{k}-(x y)^{m} g_{k}=$ $g_{k}$, for each $k=2,3, \cdots, t$. Hence, we have $\operatorname{Cay}\left(D_{2 n}, S\right)=t C_{2 m}=\frac{n}{m} C_{2 m}$ as desired. If $n$ is even ,then we have the possibility that $x=a^{\frac{n}{2}}$ and $y=a^{i} b$, then we can follow the same method as above and again we get $\operatorname{Cay}\left(D_{2 n}, S\right)=t C_{2 m}=\frac{n}{m} C_{2 m}$.

\section{Cayley Graphs of Dihedral Groups of Valency 3.}

In this section, we investigate the graph structure of $\operatorname{Cay}\left(D_{2 n}, S\right)$, whenever $|S|=3$. Let us start with the following special case.

Theorem 3.1. Let $D_{2 n}=<a, b \mid a^{n}=b^{2}=e, b a b=a^{-1}>$ be a dihedral group of order $2 n \geq 6$ and $S=\left\{a, a^{-1}, b\right\}$. Then $\operatorname{Cay}\left(D_{2 n}, S\right)=K_{2} \square C_{n}$.

Proof. Assume that $D_{2 n}=\left\{e, a, a^{2}, a^{3}, \cdots, a^{n-1}, b, a b, a^{2} b, \cdots, a^{n-1} b\right\}$. Then, we can easily see that $\left(a^{i} b\right)\left(a^{i+1} b\right)^{-1}=a^{-1},\left(a^{n-i}\right)\left(a^{n-i-1}\right)^{-1}=a$ and $\left(a^{i} b\right)\left(a^{n-i}\right)^{-1}=b$ for all $i=0,1,2, \cdots, n-1$. Hence, we will get the following graph (see figure 1 ) which is a Cartesian product of complete graph $K_{2}$ and cycle graph $C_{n}$ of length $n$. Thus $\operatorname{Cay}\left(D_{2 n}, S\right)=K_{2} \square C_{n}$ as desired.

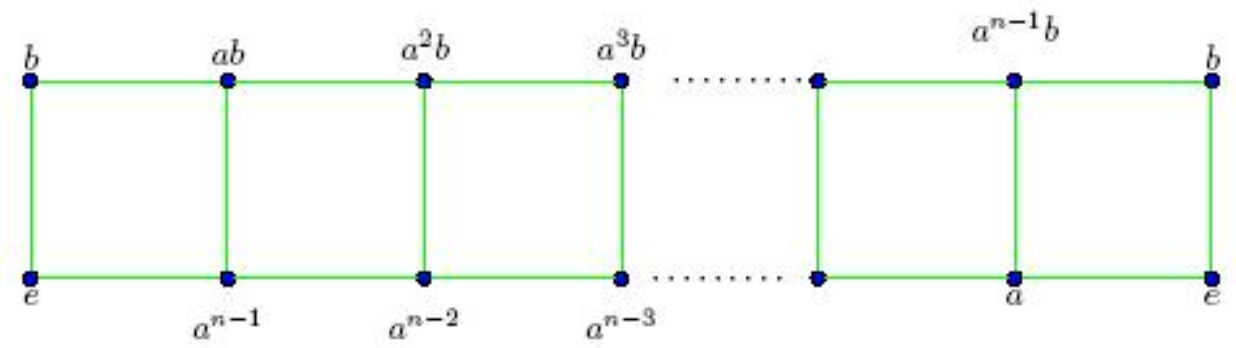

Figure 1: $\operatorname{Cay}\left(D_{2 n}, S\right)=K_{2} \square C_{n}$

Now, we are going to consider the general case that $S=\left\{x, x^{-1}, y\right\} \subseteq$ $D_{2 n}$, where $x \neq x^{-1}, y^{2}=e$. In the following theorem, we prove that $\operatorname{Cay}\left(D_{2 n}, S\right)$ with the above assumption is again $K_{2} \square C_{n}$ as similar as theorem 3.1. 
Theorem 3.2. Assume that $S=\left\{x, x^{-1}, y\right\} \subseteq D_{2 n}$, such that $x \neq x^{-1}$ and $y^{2}=e$ and $o(x)=m$. Then $\operatorname{Cay}\left(D_{2 n}, S\right)=\frac{n}{m}\left(K_{2} \square C_{m}\right)$.

Proof. Let $D_{2 n}=\left\{e, a, a^{2}, a^{3}, \cdots, a^{n-1}, b, a b, a^{2} b, \cdots, a^{n-1} b\right\}$ and $S=\left\{x, x^{-1}, y\right\}$ is a subset of $D_{2 n}$ of size 3. Since, $x \neq x^{-1}$, so $o(x)=m>2$ which implies that $x$ can not be as the form $a^{i} b$, for all $0 \leq i \leq n-1$. Thus $x=a^{i}$ for some $0 \leq i \leq n-1$ and we have $m=o(x)=o\left(a^{i}\right)=\frac{n}{(i, n)}$. Similarly, $y$ is as the form $a^{j} b$ for some $0 \leq j \leq n-1$ or possibly $a^{\frac{n}{2}}$ when $\mathrm{n}$ is even. To prove the theorem, we need the following three steps.

Step 1: Assume that $H=\langle x\rangle$ is a cyclic subgroup of order $m$. Then $H=\left\{e, x, x^{2}, \cdots, x^{m-1}\right\}$ consists a cycle graph of length $m$ as the following

$e-x-x^{2}-x^{3}-\cdots-x^{m-2}-x^{m-1}-e$. The proof is obvious.

Step 2 : Let $H z$ be a right coset of $H$ in $D_{2 n}$, where $z \notin H$. Then it consists the following cycle graph of length $m: z-x z-x^{2} z-x^{3} z-\cdots-$ $x^{m-2} z-x^{m-1} z-z$. Because $\left(x^{i} z\right)\left(x^{i+1} z\right)^{-1}=x^{-1} \in S$ for all $i=0,1,2, \cdots, n-1$.

Step 3: Suppose that $H z$ and $H w$ are two distinct right cosets of $H$ in $D_{2 n}$. If $z$ is adjacent to $w$ in $\operatorname{Cay}\left(D_{2 n}, S\right)$, then $H z \cup H w$ is produced an induced subgraph of $\operatorname{Cay}\left(D_{2 n}, S\right)$ isomorphic to the Cartesian product $K_{2} \square C_{m}$. One can easly check the proof by the corresponding graph as in figure 2 .

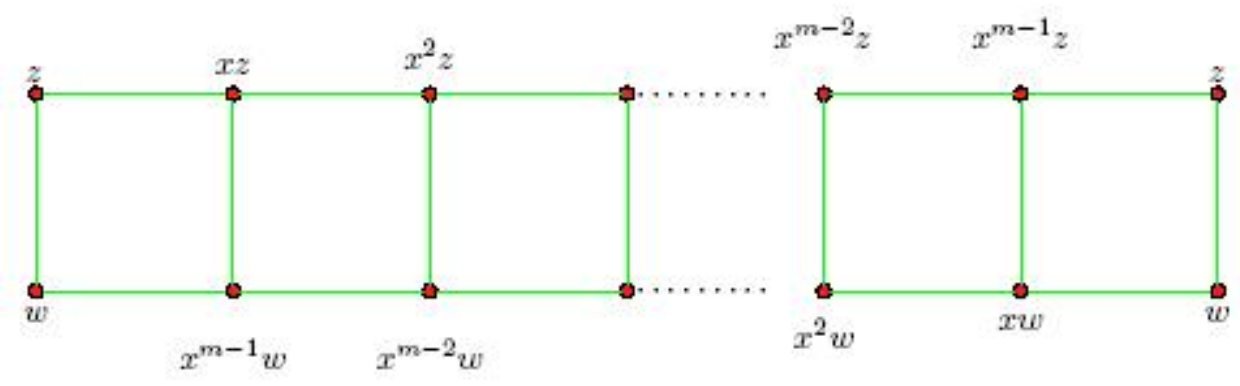

Figure 2: $K_{2} \square C_{m}$ 
Now by the above three steps, we can continue our proof of the theorem. Assume that $\left[D_{2 n}: H\right]=t$, then $\frac{2 n}{m}=t$ and so $t$ is even. We know that $\operatorname{Cay}\left(D_{2 n}, S\right)$ is 3-regular and it implies that every pair of cosets $(\mathrm{Hz}, \mathrm{Hw})$ will produce a Cartesian product $K_{2} \square C_{m}$. Since, we have $\frac{t}{2}$ disjoint pairs of such cosets, so we will have union of $\frac{n}{m}$ Cartesian product $K_{2} \square C_{m}$. Hence $\operatorname{Cay}\left(D_{2 n}, S\right)=\frac{n}{m}\left(K_{2} \square C_{m}\right)$ and the proof is complete.

Example 3.3. For Dihedral group $D_{12}$ of order 12, we have $D_{12}=<a, b \mid$ $a^{6}=b^{2}=e, b a b=a^{-1}>=\left\{e, a, a^{2}, a^{3}, a^{4}, a^{5}, b, a b, a^{2} b, a^{3} b, a^{4} b, a^{5} b\right\}$. If $S=\left\{x, x^{-1}, y\right\} \subset D_{12}$, where $x=a^{2}$ and $y=a b$. Then by Theorem 3.2 , we can see that $\operatorname{Cay}\left(D_{12}, S\right)=2\left(K_{2} \square C_{3}\right)$. Because, we have $o(x)=$ $o\left(a^{2}\right)=3, H=\langle x\rangle=\left\{e, a^{2}, a^{4}\right\}$ and distinct cosets $H a=\left\{a, a^{3}, a^{5}\right\}$, $H b=\left\{b, a^{2} b, a^{4} b\right\}$ and $H a b=\left\{a b, a^{3} b, a^{5} b\right\}$. For more details we refer to figure 3 .
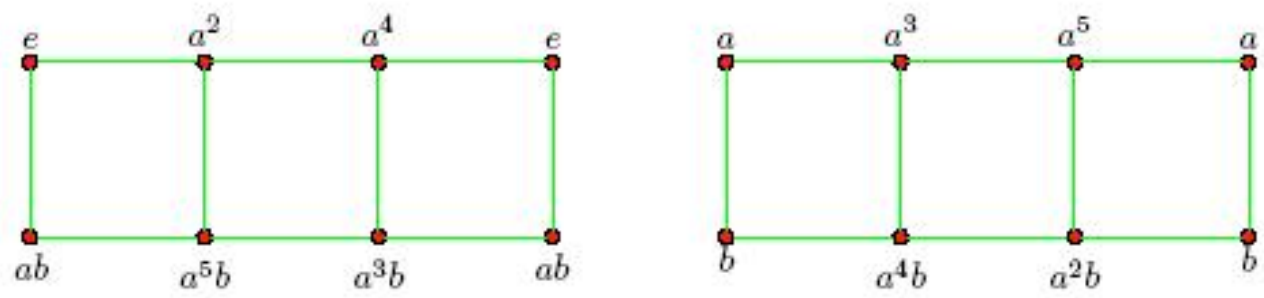

Figure 3: $\operatorname{Cay}\left(D_{12}, S\right)=2\left(K_{2} \square C_{3}\right)$

The following example is another possibility for $S \subseteq D_{2 n}$ with $|S|=3$. In fact, $S=\{x, y, z\}$, where $x^{2}=y^{2}=z^{2}=e$.

Example 3.4. Let $D_{8}$ be a dihedral group of order 8. Then we have $D_{8}=<a, b \mid a^{4}=e=b^{2}, b a b=a^{-1}>=\left\{e, a, a^{2}, a^{3}, b, a b, a^{2} b, a^{3} b\right\}$. If $S=\left\{b, a b, a^{2} b\right\}$, then $C a y\left(D_{8}, S\right)$ is the Cartesian product $K_{2} \square C_{4}$ as the following: 


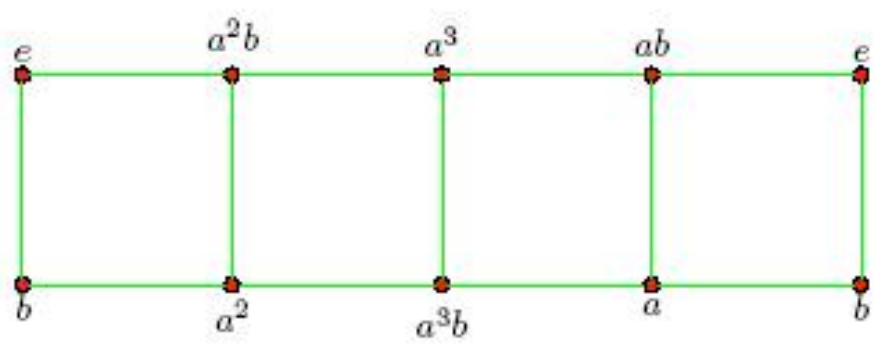

Figure 4: $K_{2} \square C_{4}$

Now by extending Theorem 2.3 and the method used in Theorem 3.2, we can state the following theorem consisting the last case of $S$ with $|S|=3$. We omit the proof.

Theorem 3.5. Let $S=\{x, y, z\}$ be a subset of $D_{2 n}$, where $n \geq 3,|S|=3$ and $x^{2}=y^{2}=z^{2}=e$. Then $\operatorname{Cay}\left(D_{2 n}, S\right)=K_{2} \square C_{n}$.

\section{References}

[1] A. Cayley, "Desiderata and suggestions: No. 2. The theory of groups: Graphical representation", American journal of mathematics, vol. 1, no. 2, pp. 174-176, 1878.

[2] L. Babai, "Automorphism groups, isomorphism, reconstruction," in Handbook of combinatorics, vol. 2, R. L. Graham, M. Grötschel, and L. Lovász, Eds. Amsterdam: Elsevier, 1995, pp. 1447-1540.

[3] V. Bafna and P. A. Pevzner, "Genome rearrangements and sorting by reversals", SIAM Journal on computing, vol. 25, no. 2, pp. 272-289, 1996.

[4] G. Erskine, "Diameter 2 Cayley graphs of dihedral groups", Discrete mathematics, vol. 338, no. 6, pp. 1022-1024, 2015.

[5] X- G. Fang, C-H. Li, and M-Y. Xu, "On edge-transitive Cayley graphs of valency four", European journal of combinatorics, vol. 25, no. 7, pp. 1107-1116, 2004. 
[6] A. Kelarev, J. Ryan and J. Yearwood, "Cayley graphs as classifiers for data mining: the influence of asymmetries", Discrete mathematics, vol. 309, no. 17, pp. 5360-5369, 2009.

[7] C. H. Li, "On isomorphisms of finite Cayley graphs-a survey", Discrete mathematics, vol. 256, no. 1-2, pp. 301-334, 2002.

[8] L. Lu, Q. Huang, and X. Huang, "Integral Cayley graphs over dihedral groups", Journal of algebraic combinatorics, vol. 47, no. 4, pp. 585-601, 2017. https:/ / doi.org/ 10.1007/ s10801-017-0787-x

[9] Š. Miklavič and P. Potočnik, "Distance-regular Cayley graphs on dihedral groups", Journal of combinatorial theory, Series B, vol. 97, no. 1, pp. 14-33, 2007.

[10] J. Pan, "Locally primitive Cayley graphs of dihedral groups", European journal of combinatorics, vol.36, pp. 39-52, 2014.

[11] D. J. S. Robinson, A course in the theory of groups. New York, NY: Springer, 1982.

[12] D. B. West, Introduction to graph theory, 2nd ed. New York, NY: Prentice Hall, 2001.

\author{
Saba AL-Kaseasbeh \\ Department of Mathematics, \\ Faculty of Science, \\ Tafila Technical University, \\ Tafila, \\ Jordan \\ e-mail: saba.alkaseasbeh@gmail.com \\ and
}

\author{
Ahmad Erfanian \\ Department of Mathematics and Center of Excellence in Analysis on \\ Algebraic Structures, \\ Ferdowsi University of Mashhad, \\ Mashhad, \\ Iran \\ e-mail: erfanian@um.ac.ir \\ Corresponding author
}

